\title{
A Mechanochemical Model for Adult Dermal Wound Contraction and the Permanence of the Contracted Tissue Displacement Profile
}

\author{
Luke Olsen†, Jonathan A. Sherratt $\ddagger$ and Philip K. Maini $\dagger$ \\ $\uparrow$ Centre for Mathematical Biology, Mathematical Institute 24-29 St Giles', Oxford, \\ $O X 13 L B, U . K$. \\ $\$$ Nonlinear Systems Laboratory, Mathematics Institute University of Warwick, \\ Coventry, CV4 7AL, U.K.
}

(Received on 19 July 1994, Accepted in revised form on 7 June 1995)

\begin{abstract}
The healing of adult mammalian skin wounds involves a complex sequence of spatially and temporally coordinated processes. Wound contraction, by reducing the size of the injury, is an intrinsic component of full-thickness excisional dermal wound healing. The underlying biomechanics of wound contraction, however, are not fully understood, and little is known about the pathogenesis of severe medical conditions known as fibrocontractive diseases. The aim of this work is to investigate a deterministic mathematical model in order to obtain insight into the mechanistic relationships between wound contraction and associated normal and pathological healing processes. The model describes the essential roles of fibroblast and myofibroblast cells, a chemical growth factor and the extracellular matrix which includes type I collagen. The model results are qualitatively consistent with the biology of fibroplasia and wound contraction. It is shown that a contracted state evolves during a (long) transient phase of healing known as "proliferation", while collagen kinetics are fundamental to the considerably longer "remodelling" phase. Some quantitative results, notably on the evolution of wound contraction, compare favourably with experimental data. Application of the model to adult human dermal wound healing in vivo, with a greater understanding of the underlying biological mechanisms involved, may suggest strategies for controlling contraction and fibrocontractive diseases.

(C) 1995 Academic Press Limited
\end{abstract}

\section{Introduction}

The healing of full-thickness skin wounds in adult mammal involves a highly complex and interdependent series of repair processes, operating within characteristic spatio-temporal intervals (Clarke, 1988). Wound contraction, the biomechanical phenomenon in which the wound boundaries are drawn inwards, is a ubiquitous and essential feature in the healing of these wounds and, together with tissue formation, normally effects full wound closure (Rudolph, 1980; Rudolph et al., 1992). The rate and extent of contraction depends upon species, age, wound dimensions and other parameters, such as infection and anatomical location (McGrath \& Hundahl, 1982; McGrath \& Simon, 1983; Rudolph, 1979; Skalli \& Gabbiani, 1988; Skalli et al., 1989). Fetal wounds, for example, are believed to heal without significant contraction or scarring (Skalli \& Gabbiani, 1988; Shah et al., 1992; Longaker et al., 1992). In adult humans, a typical excisional wound may undergo a $20-30 \%$ reduction in wound area because of contraction over a period of several weeks, while in other mammals the rate and extent of 
contraction is generally greater [up to $80-90 \%$ of wound closure is attributed to contraction in rats; Rudolph (1979) McGrath \& Simon (1983)].

By reducing the size of the defect, wound contraction is usually beneficial to the overall repair process. However, insufficient contraction may cause delayed or impaired healing, whilst excessive contraction often induces poor quality repair with substantial scarring (Skalli \& Gabbiani, 1988; Jennings \& Hunt, 1992; Rudolph et al., 1992; Shah et al., 1992). Healing abnormalities occurring at the level of wound contraction may also result in fibrocontractive diseases, many of which are clinically severe, physically undesirable and functionally debilitating (see, for review, Rudolph, 1980; Skalli \& Gabbiani, 1988; Rudolph \& Vande Berg, 1991; Skalli et al., 1989). Despite intensive research, the underlying biological mechanisms of wound contraction and of associated pathologies remain incompletely understood (Gabbiani, 1992; Rudolph et al., 1992).

The aim of this work, therefore, is to develop a mathematical model for the fundamental cell biology and biomechanics of wound contraction, in order to predict the behaviour of experimental and clinical wounds and to yield insight into the pathogenesis of fibrocontractive diseases.

An effective deterministic model must predict steady states representing normal skin, a contracted wound and fibrocontractive diseases. The initial injury is a large perturbation of the normal, unwounded state (Skalli \& Gabbiani, 1988), with the ensuing healing process encapsulated by the evolution of the model dynamical system. The precise sense, however, in which the contracted state is a "steady solution" of the system, is vital in elucidating the underlying biological mechanisms of normal and pathological wound contraction, and has attracted recent modelling debate (Murray et al., 1988; Tranquillo \& Murray, 1992). Thus, the emphasis of this paper is on investigating the model to acquire a greater understanding of the contracted wound state. A subsequent report will address pathological transitions of fibrocontractive disease states as predicted by the model discussed below (L. Olsen et al., submitted).

\section{A Biological Overview of Dermal Wound Healing}

Adult mammalian skin is composed of a complex array of local and systemic elements, each providing important contributions towards the regulatory and protective functions of the skin (Odland, 1991; Mast, 1992). Anatomically, the skin is partitioned into two layers (Fig. 1). The outermost layer is the epidermis, consisting of densely packed keratinocyte cells which continually proliferate and differentiate (Dover \& Wright, 1991). The underlying, thicker layer is known as the dermis and is composed of fibroblast cells, extracellular matrix (ECM), blood capillaries and other circulatory, secretory and structural components (Odland, 1991; Mast, 1992). Fibroblasts perform many vital roles in dermal wound healing and are central to contraction (Clark, 1988, 1993; Rudolph et al., 1992). The various fibrous and soluble molecules which constitute the ECM include proteins such as collagen - the most abundant solid-phase component of the skin, and enzymes which enable the ECM to undergo constant turnover (Odland, 1991). Beneath the dermis, but not clearly demarcated from it, are subcutaneous strata of fatty, muscular and fibrous tissues, interspersed with circulatory vessels (Mast, 1992).

The epidermal response to wounding is well documented, both biologically (Clark, 1989; Oldland \& Ross, 1968) and mathematically (Sherratt \& Murray, 1992; Sherratt et al., 1992). Full-thickness dermal wound healing, however, is required when epidermal and dermal tissues are excised (Fig. 1). The multitude of interregulatory dermal repair processes, of which wound contraction is a prominent and important consequence, have attracted enormous biomedical interest (see, for review, Clark, 1989; Jennings \& Hunt, 1992; Mast, 1992; Rudolph et al., 1992). These processes may be grouped into three overlapping temporal phases: inflammation, proliferation and remodelling (Clark, 1988).

Platelets from damaged blood vessels initiate the inflammatory phase within a few hours of wounding by aggregating to form a stable clot, thereby sealing the wound from further damage or infection (Clark, 1989). They also release a variety of biochemical substances, including several types of growth factors which individually and synergistically regulate many repair processes (Sprugel et al., 1987; Clark, 1991; Grotendorst, 1992; Martin et al., 1992). Notably, they attract inflammatory cells into the wound site, triggering further biochemical and enzymatic cascades which initiate the following phases of healing (Clark, 1989).

The proliferative phase usually begins several days post-wounding. Epidermal integrity is re-established as keratinocytes divide and migrate above the wound tissue (Odland \& Ross, 1968). Simultaneously, angiogenesis takes place, whereby new blood capillaries grow into the wound tissue from surrounding vessels, thus establishing a blood supply and hence metabolic regulation in the wound (Arnold \& West, 1992; Whalen \& Zetter, 1992). In addition, growth 


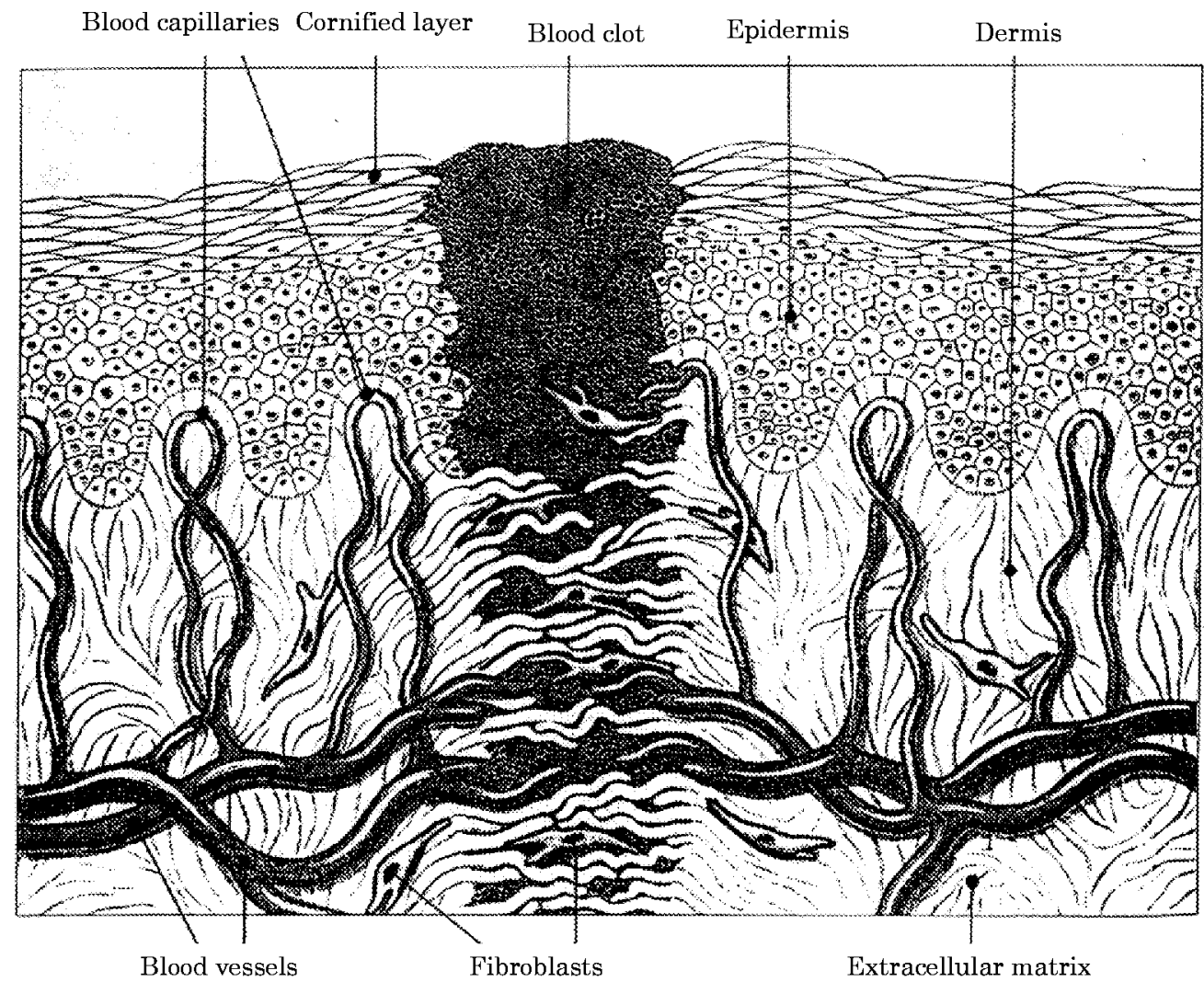

Blood vessels

Fibroblasts

Extracellular matrix

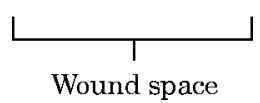

FIG. 1. A schematic representation of a section through wounded adult mammalian skin. Note that, in reality, a typical full-thickness dermal excisional wound is an order of magnitude greater in width than in depth. Subcutaneous tissues are not shown. Adapted from Asmussen \& Söllner (1993)

factors chemotactically recruit fibroblasts into the wound from adjacent dermis (Grotendorst, 1992) and stimulate these cells to proliferate and to produce growth factors and ECM molecules, including collagen (Clark, 1993). Collectively, these processes are termed "fibroplasia". Some fibroblasts are also phenotypically converted into myofibroblasts, which contain intracellular actin stress fibres and other molecular and structural components characteristically associated with contractile cells (Gabbiani et al., 1973; Skalli \& Gabbiani, 1988; Gabbiani, 1992). Myofibroblasts are vitally implicated in wound contraction (Gabbiani et al., 1971; Rudolph, 1979; Skalli \& Gabbiani, 1988, Rudolph et al., 1992). As fibroblasts move through the wound tissue, they exert traction forces on collagen fibres in the ECM (Stopak \& Harris, 1982; Oster et al., 1983; Ehrlich \& Rajaratnam, 1990). The resultant tension is transmitted throughout the wound space by the
myofibroblast/ECM network in which ECM fibrillar assemblies are associated with intracellular stress fibres at specific locations on the myofibroblast cell surfaces (Singer et al., 1989). This synchronised transduction of the cell traction forces culminates in wound contraction (Skalli \& Gabbiani, 1988; Rudolph et al., 1992).

ECM remodelling typically proceeds for many months after fibroplasia and contraction have ceased, normally three or four weeks after injury (Rudolph, 1979; McGrath \& Simon, 1983; Clark, 1989). Although inflammatory cell and myofibroblast levels will have receded, fibroblasts continue to perform essential roles in reorganizing the ECM in order to restore the biological and mechanical resilience of the wound tissue (Snowden \& Cliff, 1985; Jennings \& Hunt, 1992).

Investigating the processes of fibroplasia and contraction is central to the aims of this work. 
Providing a framework in which to model (from experimental data) these components of the proliferative phase of healing is therefore the focus of the following section.

\section{Modelling Wound Contraction}

The model is based upon a fundamental conservation law for each space- and time-dependent quantity, $Q=Q(x, t)$ :

$$
\frac{\partial Q}{\partial t}=-\nabla \cdot \mathbf{J}_{Q}+f_{Q},
$$

where $\mathbf{J}_{Q}$ is the flux of $Q$ and $f_{Q}$ are the kinetic terms (rates of production and loss of $Q$ ).

The fibroblast density, myofibroblast density, collagen concentration and the concentration of a "generic" growth factor are modelled as distinct variables, thereby accounting for the important cell biology of fibroplasia and wound contraction (Section 2). Multiple regulatory chemicals are known to mediate these processes in reality (Clark, 1989, 1991), but for simplicity we model a single growth factor which promotes the healing response. Plateletderived growth factor (PDGF) exhibits much of the requisite behaviour of the generic chemical (Ross et al., 1986; Huang et al., 1988; Raines et al., 1990; Grotendorst, 1992; Lepistö, 1992), and experimental data for PDGF are used to determine the relevant parameter values (see Appendix). The explicit inclusion of fibroblasts and myofibroblasts as distinct cell types is a new modelling approach, enabling the fundamental cellular aspects of wound contraction (Section 2) and of fibrocontractive diseases to be more accurately described (Skalli \& Gabbiani; 1988; Skalli et al., 1989).

The complex biomechanics of wound contraction has recently posed a considerable and important modelling challenge (McGrath \& Simon, 1982; Murray et al., 1988, Tranquillo \& Murray, 1992). Following previous mechanochemical models for morphogenesis, we consider the conservation of tissue momentum (Murray et al., 1983; Murray, 1989). This yields the "force-balance" equation which governs the tissue displacement - an obvious quantitative measure of wound contraction (Murray et al., 1988).

The model variables to represent the fibroblast density, $n$, myofibroblast density, $m$, chemical concentration, $c$, collagen concentration, $\rho$, and tissue displacement, $\mathbf{u}$, satisfy the following conservation equations. The fluxes and kinetics model the relevant processes involved in fibroplasia and wound contraction (Section 2), with fundamental forms derived from experimental and theoretical results (see references below).

$$
\text { FIBROBLASTS, } n(\mathbf{x}, t)
$$

$$
\begin{aligned}
\frac{\partial n}{\partial t} & =\nabla \cdot\left[D_{n} \nabla n-\frac{a_{n}}{\left(b_{n}+c\right)^{2}} n \nabla c-n \frac{\partial \mathbf{u}}{\partial t}\right] \\
& +\left(r_{n}+\frac{r_{n_{\max }} c}{C_{1 / 2}+c}\right) n\left(1-\frac{n}{K}\right) \\
& -\frac{k_{\operatorname{lax}_{\max }} c}{C_{k}+c} n+k_{2} m-d_{n} n .
\end{aligned}
$$

The contributions to the cell flux are: random dispersal (Bard \& Hay, 1975), modelled by linear Fickian diffusion - a standard representation of random cell motion (Murray, 1989); growth factormediated chemotaxis (Grotendorst, 1992; Seppä et al., 1992), where the chemotactic sensitivity function originates from a biologically realistic cell surface receptor mechanism (Sherratt et al., 1993); and passive convection by the moving tissue. For the kinetics, there is: mitosis, using a logistic form for the cell proliferation rate, in quantitative agreement with experimental data on fibroblast growth curves (Vande Berg et al., 1989) with growth factor enhancement of the linear growth rate (Raines et al., 1990); phenotypic transformation into myofibroblasts, stimulated by growth factors (Skalli \& Gabbiani, 1988; Gabbiani; 1992); a source term from the reverse phenotypic transformation; and natural cell death (Alberts et al., 1990).

$$
\begin{gathered}
\text { MYOFibroblasts, } m(\mathbf{x}, t) \\
\frac{\partial m}{\partial t}=\nabla \cdot\left[-m \frac{\partial \mathbf{u}}{\partial t}\right]+\epsilon_{r}\left(r_{n}+\frac{r_{n_{\max }} c}{C_{1 / 2}+c}\right) m\left(1-\frac{m}{K}\right) \\
+\frac{k_{1_{\max }} c}{C_{k}+c} n-k_{2} m-d_{m} m .
\end{gathered}
$$

Without evidence that myofibroblasts are actively motile, passive convection is taken to be the only flux. The kinetics correspond to those for fibroblasts: namely mitosis, phenotypic transformation and cell death.

$$
\begin{gathered}
\text { CHEMICAL, } c(\mathbf{x}, t) \\
\frac{\partial c}{\partial t}=\nabla \cdot\left[D_{c} \nabla c-c \frac{\partial \mathbf{u}}{\partial t}\right]+\frac{(p-1) k_{p}^{\prime}\left(n+\zeta_{p} m\right) c}{\Gamma_{p}+c} \\
-\frac{k_{c}^{\prime}\left(n+\zeta_{c} m\right) c}{\Gamma_{c}+c}-d_{c} c .
\end{gathered}
$$


The chemical diffuses through the ECM (assuming a constant diffusion coefficient) and is convected by the tissue. The kinetics involve production and consumption of the chemical by both fibroblasts and myofibroblasts - the roles of cell surface receptor molecules (Raines et al., 1990; Sato et al., 1993) again suggest the functional forms for these processes (see Appendix for details). There is also natural decay of the chemical, with a tissue clearance half-life of only a few minutes (Bowen-Pope et al., 1984; Huang et al., 1988).

$$
\begin{gathered}
\text { COLLAGEN, } \rho(\mathbf{x}, t) \\
\frac{\partial \rho}{\partial t}=\nabla \cdot\left[-\rho \frac{\partial \mathbf{u}}{\partial t}\right]+\left(r_{\rho}+\frac{r_{\rho_{\max }} c}{C_{\rho}+c}\right) \frac{n+\eta_{b} m}{R_{\rho}^{2}+\rho^{2}} \\
-d_{\rho}\left(n+\eta_{d} m\right) \rho .
\end{gathered}
$$

Collagen fibres are generally linked in a mesh-like network (Clark, 1989; Jennings \& Hunt, 1992), so passive convection is the only contribution to the collagen flux. For the kinetics, there is: synthesis by both fibroblasts and myofibroblasts, with growth factor enhancement (Clark, 1989; Pierce et al., 1991); and enzymatic degradation by both cell types (Uitto, 1984; Skalli \& Gabbiani, 1988; Jeffrey, 1992).

$$
\begin{aligned}
& \text { DISPLACEMENT, } \mathbf{u}(\mathbf{x}, t) \\
& 0=\nabla \cdot\left[\mu_{1} \frac{\partial \epsilon}{\partial t}+\mu_{2} \frac{\partial \theta}{\partial t} \boldsymbol{I}+E^{\prime}\left(\epsilon+v^{\prime} \theta \boldsymbol{I}\right)\right. \\
& \left.+\frac{\tau_{0}(1+\xi m) n \rho}{R_{\tau}^{2}+\rho^{2}} \boldsymbol{I}\right]-s \rho \mathbf{u} .
\end{aligned}
$$

Here, $\epsilon=\frac{1}{2}\left(\nabla \mathbf{u}+\nabla \mathbf{u}^{T}\right)$ is the strain tensor and $\theta=\nabla \cdot \mathbf{u}$ is the dilation. Following previous mechanochemical models of tissue morphogenesis proposed by Murray and colleagues (Murray \& Oster, 1984; Murray et al., 1988), two contributions of the flux of momentum are included. First, there are traction stresses exerted on collagen fibres in the ECM by fibroblasts at their cell surfaces. These stresses are modelled as the unit tensor multiplied by an isotropic traction coefficient, taken to be proportional to the fibroblast density and to the collagen concentration (Murray et al., 1988). Enhancement of the traction force by myofibroblasts and inhibition at high collagen densities (those typical of unwounded dermis, for example,) are assumed. Second, there are intrinsic ECM stresses associated with tissue deformation. Using the simplifying assumptions of tissue isotropy, a simple viscoelastic rheology and a linear stress-strain/strain rate depen- dence (valid for small strains and strain rates), a constitutive relation for the ECM stress tensor is derived, as in other mechanical models (Landau \& Lifshitz, 1970; Fung, 1981; Murray, 1989; Tranquillo \& Murray, 1992). The only "kinetics" are due to the body forces exerted on the tissue by fibrous ECM attachments between the dermal tissue and subcutaneous layers. These are modelled simply as linear restoring forces acting against tissue displacement and are taken to be proportional to the displacement (per unit of fibrous ECM) (Murray et al., 1992; Tranquillo \& Murray, 1992).

Together with negligible inertial effects owing to the high tissue viscosity, these simplifications yield the "force balance" equation (5) above. Despite its apparent complexity, no simpler description of the biomechanics of wounded or normal dermal tissue has been formulated. Indeed, the assumptions with respect to the tissue rheology and to the constitutive relation have provoked ongoing modelling debate on the biomechanical detail necessary to derive a realistic force balance equation.

\section{THE NONDIMENSIONAL 1-D LINEAR WOUND MODEL}

In reality, wound healing occurs in a three-dimensional domain. However, the thickness of the adult skin (approximately $1-2 \mathrm{~mm}$ ) is much smaller than the typical dimensions of the excisional wounds from which experimental data has been collated and to which this model is applicable (generally between 1 and $5 \mathrm{~cm}$ ). Thus, the skin may be regarded as two-dimensional (Fung, 1981). In this paper, we restrict attention to the simple cases of (long) rectangular and circular wounds, represented by linear and axisymmetric geometries as illustrated in Fig. 2, so that the problem is one-dimensional.

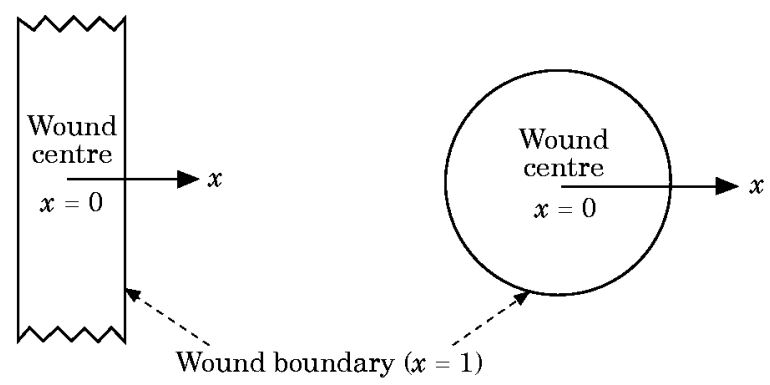

Linear model Axisymmetric model

FIG. 2. Diagram of the linear and axisymmetric wound geometries in the model (in the plane of the skin), with $x$ denoting the distance from the wound centre, $x=0$. The initial wound boundaries are at (normalized) distance unity from the wound centre, as shown. 
To clarify the roles of the parameters in the model, we nondimensionalize the variables and the parameters (see Appendix for details) to obtain the five nondimensional model equations for the 1-D linear wound (omitting the tildes for notational simplicity):

$$
\begin{aligned}
& \frac{\partial n}{\partial t}=\frac{\partial}{\partial x}\left[D_{n} \frac{\partial n}{\partial x}-\frac{\alpha}{(\beta+c)^{2}} n \frac{\partial c}{\partial x}-n \frac{\partial u}{\partial t}\right] \\
& +\sigma\left(1+\frac{A c}{B+c}\right) n(1-\gamma n) \\
& -\frac{\kappa_{1} c}{C+c} n+\kappa_{2} m-\delta n \\
& \frac{\partial m}{\partial t}=\frac{\partial}{\partial x}\left[-m \frac{\partial u}{\partial t}\right]+\epsilon_{\sigma} \sigma\left(1+\frac{A c}{B+c}\right) m(1-\gamma m) \\
& +\frac{\kappa_{1} c}{C+c} n-\kappa_{2} m-\lambda \delta m \\
& \frac{\partial c}{\partial t}=\frac{\partial}{\partial x}\left[D_{c} \frac{\partial c}{\partial x}-c \frac{\partial u}{\partial t}\right]+\frac{\kappa_{c}(n+\zeta m) c}{\gamma_{c}+c}-\delta_{c} c \\
& \frac{\partial \rho}{\partial t}=\frac{\partial}{\partial x}\left[-\rho \frac{\partial u}{\partial t}\right]+\left[\omega\left(1+\frac{P c}{Q+c}\right)\right. \\
& \left.\times \frac{1}{\phi^{2}+\rho^{2}}-\delta_{\rho} \rho\right](n+\eta m) \\
& 0=\mu \frac{\partial^{3} u}{\partial x^{2} \partial t}+\frac{\partial^{2} u}{\partial x^{2}}+v \frac{\partial}{\partial x}\left[\frac{(1+\xi m) n \rho}{\psi^{2}+\rho^{2}}\right]-s \rho u
\end{aligned}
$$

Even after non-dimensionalization, the model contains 31 parameters. Accurate estimation of the values of these parameters is vital before the model can be usefully applied, and our methods of parameters value estimation are discussed in detail in the Appendix.

Equations (6-10) are solved in the 1-D linear domain $x>0$, with symmetric boundary conditions at $x=0$. The spatial coordinate is rescaled so that the initial wound space is defined as $0 \leqslant x \leqslant 1$ (Fig. 2). The wound is assumed to be sufficiently isolated from other large perturbations of normal dermis, so that at infinity, the model variables assume their constant, dermal values (which are calculated in the next section).

The initial conditions $(t=0)$ are defined at the onset of the proliferative phase of healing (Section 2). There are no cells inside the wound initially, and the dermal level of fibroblasts outside, $n=1$ (see Appendix). The initial myofibroblast density is zero everywhere. The initial chemical profile, $c_{\text {init }}$, is due to the supply of growth factors during the inflammatory phase (Clark, 1988; Jennings \& Hunt, 1992; Martin et al., 1992), and assumes a form that decreases smoothly to zero around the wound margins owing to diffusion. Specifically, we take $c_{\text {init }}(x)=1 /\left(1+x^{4}\right)$; the model results, however, are not sensitive to minor alterations of the form chosen. The collagen concentration is at some small value, $\rho_{\text {init }} \ll 1$, inside the wound (due to low-level depositions of ECM molecules by infiltrating cells such as macrophages during the early, inflammatory phase of healing), and the dermal value, $\rho=1$ outside. $\rho_{\text {init }}$ must be positive, however, to avoid difficulties with the numerical simulations. Finally, the displacement is zero everywhere.

\section{Predictions of the Model}

\subsection{STEADY STATES}

The general problem of spatially inhomogeneous steady states is analytically intractable. Motivated however, by the biological requirement that the model exhibits a locally stable spatially inhomogeneous steady state which represents unwounded dermis, the uniform case is considered first, as given by the system of algebraic equations obtained by setting the derivatives in (6-10) to zero.

From (10), either $\rho=0$ or $u=0$. In the case $\rho=0$, the kinetic terms of (8) and (9) imply that $n, m$ and $c$ are also zero, yielding a trivial solution. This is of little biological interest, since it is not relevant to any realistic steady state. If $u=0$, then there is no tissue displacement when the model variables are at a uniform steady state, in agreement with the boundary condition $u(0, t)=0$.

In addition, normal human dermis produces significant concentrations of active growth factors only in response to external stimuli such as wounding (Clark, 1991; Martin et al., 1992). Therefore, investigations are restricted here to steady states for which $c=0$. The residual, simplified, uniform steady-state equations are solved explicitly, with the following results (see Table 1). Here "existence" means the requirement that the species variables $n, m$, $c$ and $\rho$ assume real, nonnegative values. Linear stability is deduced from the standard analysis which yields specific parameter inequalities, with which the parameter values suggested by our estimates (see Appendix) are compared.

The scalings chosen to nondimensionalize the fibroblast density, $n$, and the collagen concentration, $\rho,\left(n_{0}\right.$ and $\rho_{0}$ respectively-see Appendix $)$ set the unwounded (normal dermal) steady state values to unity. $n_{+}$and $m_{+}$may be calculated analytically from 
(6) and (7). The finding that $n_{+}>1$ and $m_{+}>0$ is indicative of healing abnormalities with excessive cellular responses and, in particular, of fibrocontractive diseases in which myofibroblasts persist (Skalli \& Gabbiani, 1988; Skalli et al., 1989; Rudolph \& Vande Berg, 1991).

\subsection{THE PROBLEM OF THE PERMANENCE OF WOUND CONTRACTION}

We assume that the phenomenon of normal wound contraction is manifested in a stable steady state in which the species variables are uniform, namely $\left[n^{*}, m^{*}, c^{*}, \rho^{*}\right]=[1,0,0,1]$, whereas the displacement is spatially varying. Under these conditions, (10) implies that the steady displacement profile, $u(x)$, satisfies the simple, linear boundary value problem:

$$
\frac{\mathrm{d}^{2} u}{\mathrm{~d} x^{2}}-s u=0, \quad u(0)=0, \quad \frac{\mathrm{d} u}{\mathrm{~d} x}(\infty)=0
$$

The only solution to (11) is $u \equiv 0$. Thus, at steady state, no non-trivial displacement profile exists when the species variables are spatially uniform. With a corresponding result for the axisymmetric case [in which (11) is a modified Bessel equation], the model does not appear to exhibit a contracted steady state. This problem is discussed further below.

\subsection{NUMERICAL SIMULATIONS}

The above analyses suggest that the system should return to the dermal steady state, of which the initial state is a large perturbation. This is confirmed numerically as shown in Fig. 3, noting that: (a) the fibroblast density, $n$, gradually reattains its dermal value; (b) myofibroblasts, $m$, appear quickly but transiently, with highest densities around the wound boundary; (c) the chemical concentration, $c$, decays rapidly to zero with diffusion from the wound site; (d) the collagen concentration, $\rho$, deviates only slightly from its initial profile over the 30-day time course of these simulations (passive convection appears to be the dominant feature here), and is restored to its dermal value over several years (not shown); (e, f) the displacement, $u$, becomes negative, most markedly just inside the wound margin. Over a considerably longer time scale of several years, however, the numerical simulations confirm that the displacement relaxes slowly back to zero (not shown).

\subsection{COMPARISONS BETWEEN THE MODEL AND EXPERIMENTAL RESULTS}

There is relatively little quantitative experimental data on the fundamental processes of fibroplasia and wound contraction in vivo. This is probably a consequence of the multitude of interacting processes that occur in real wound healing, which render measurement of the individual variables a difficult task. Although this is untrue of in vitro models, no single experimental system is at present regarded as a realistic simulator of the proliferative phase of wound healing. Hence, we consider the few sources of available experimental data which can be used to assess the qualitative predictive abilities of our model.

The spatiotemporal evolution of the tissue displacement is of particular interest. McGrath \& Simon (1983) analysed the rate of change of the area of full-thickness dermal circular excisional wounds in rats (as a fraction of the wound area prior to contraction). Their experimental data and the numerical simulations of the axisymmetric model are compared in Fig. 4; here we use the model without collagen kinetics, since a "final contracted area" can be calculated for this case (see Fig. 4 legend and Section 4.5). Quantitatively, the two sets of data are similar, but not perfectly correlated. Contraction appears to be slightly faster in the experimental data between 1 and 2 weeks after wounding. As the authors noted, however, inaccuracies in wound area measurements are likely during this period because epidermal regeneration may hinder the precise calculation of the positions of the dermal wound

TABLE 1.

Analysis of spatially uniform steady states (with $c=0$ ) listing the parametric constraints for existence and local stability of each steady state

\begin{tabular}{lccc}
\hline & \multicolumn{2}{c}{ Spatially uniform steady states (with $c=0)$} & \\
\hline$[n, m, c, \rho, u]:$ & {$[0,0,0, \rho, 0]$} & {$[1,0,0,1,0]$} & {$\left[n_{+}, m_{+}, 0,1,0\right]$} \\
Interpretation: & "Trivial" & "Normal dermal" & $\kappa_{2}+\lambda \delta<\epsilon_{\sigma} \sigma$ \\
"Existence" conditions: & Always exists & $\delta<\sigma$ & $\delta<\sigma$ \\
Stability conditions: & $\sigma<\delta$ & $\delta<\sigma$ & $\kappa_{2}+\lambda \delta<\epsilon_{\sigma} \sigma$ \\
(deduced from linear & $\epsilon_{\sigma} \sigma<\kappa_{2}+\lambda \delta$ & $\epsilon_{\sigma} \sigma<\kappa_{2}+\lambda \delta$ & $\kappa_{c}\left(n_{+}+\zeta m_{+}\right)<\gamma_{c} \delta_{c}$ \\
stability analysis) & & $\kappa_{c}<\gamma_{c} \delta_{c}$ & Unstable \\
$\begin{array}{l}\text { Stability result: } \\
\text { (for parameter values }\end{array}$ & Stable & \\
as in Fig. 3 legend) & & & \\
\hline
\end{tabular}



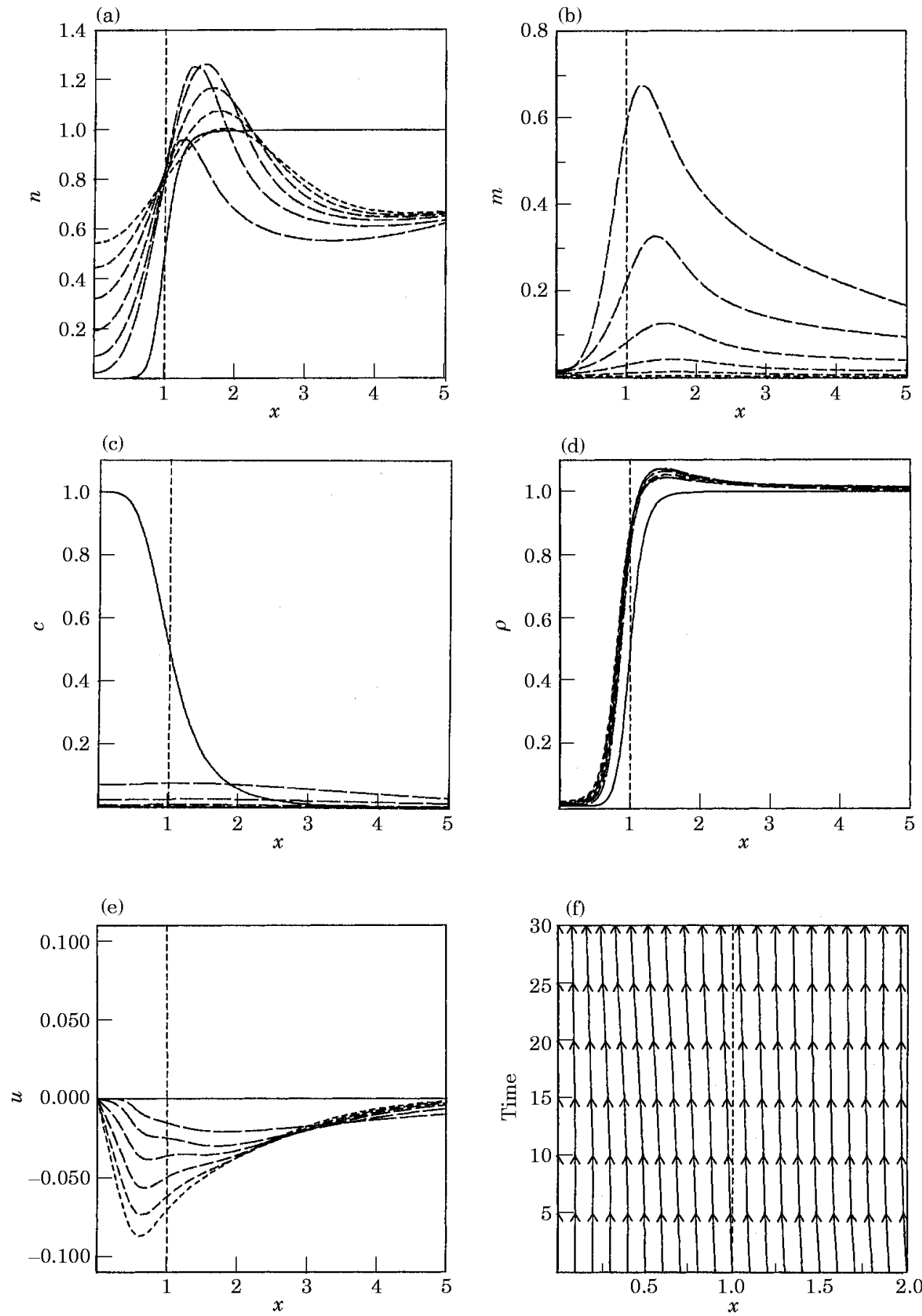

FIG. 3. Numerical simulations of the linear wound model, using the method of lines and Gear's method, showing the evolution of (a) fibroblast cell density, (b) myofibroblast cell density, (c) chemical concentration, (d) collagen concentration, and (e) tissue displacement profiles, plotted against the distance from the wound centre at sucessive time intervals of $\Delta t=5$ (days). In (f), the simulated movement of tattoo marks located across the wound and adjacent dermis is shown. A finite domain approximation, $x_{\infty}=10$, is used for these simulations, so zero flux conditions are imposed at this boundary rather than constant, dermal values at infinity. Parameter values are: $D_{n}=0.02, \alpha=0.5, \beta=0.2, \sigma=0.02, A=44.5, B=1, \gamma=0.01, \kappa_{1}=10, C=1, \kappa_{2}=1, \delta=0.0198, \epsilon_{\sigma}=0.5, \lambda=10, D_{c}=1.2, \kappa_{c}=0.4, \gamma_{c}=1$, $\delta_{c}=0.5, \zeta=1, \omega=0.008, P=10, Q=0.1, \phi=3, \delta_{\rho}=0.0008, \eta=2, \mu=20, v=0.02, \xi=10, \psi=0.005, s=1, \rho_{\text {init }}=0.01$.

margins. The results are very similar over the 30-day time-course of the simulations in the case where the collagen kinetics are included (not shown). Other experimental data on the area of the contracting wounds (Rudolph, 1979; McGrath \& Hundahl, 1982; Rudolph et al., 1992) are also in close agreement 
with the numerical simulations of the model (not shown).

Another useful indicator for the quantitative accuracy of the model is the chemical dynamics, since the growth factors regulate many vital aspects of the proliferative phase of wound healing (Sections 2 and 3). The assay of Sprugel et al. (1987), in which small porous chambers were subcutaneously implanted into rats, was aimed at providing a quantitative method of assessing fibroplasia in vivo. The "wound chambers" contained a growth factor/matrix medium believed to be an approximate representation of the wound state late in the inflammatory phase of healing (Section 2), when growth factors and a provisional wound matrix have been deposited to stimulate fibroplasia. For comparison with our model, the clearance data on PDGF are used, since this growth factor is a vital stimulant for fibroplasia, and the roles of the generic chemical in our model are primarily modelled on those of PDGF (Section 3 and Appendix). The model predictions for both the linear and circular wounds are shown in Fig. 5, together with the experimental data of Sprugel et al. (1987). In addition to close correlations, the stimulated clearance curves exhibit the same "biphasic" qualitative properties of those from the experimental data (Sprugel et al., 1987). The initial, rapid decline in chemical levels

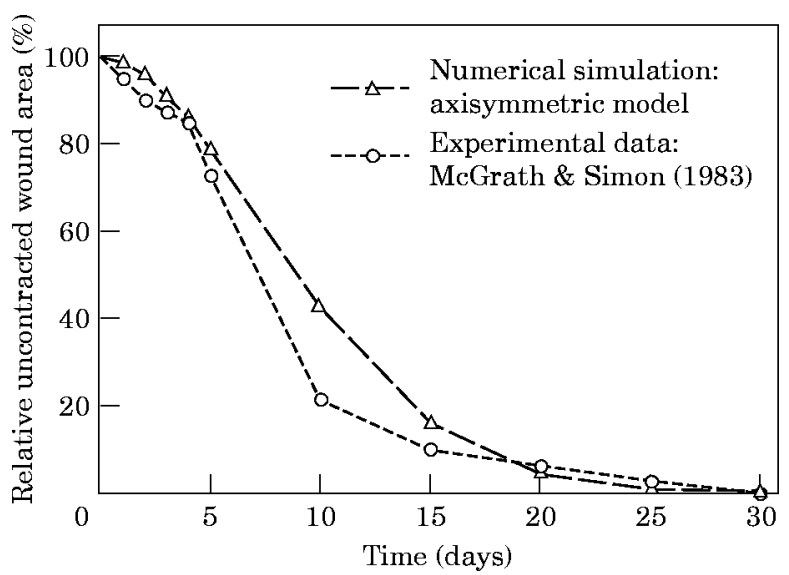

FIG. 4. Simulated and experimental data for wound contraction, plotted as the difference between the current and the fully contracted wound area divided by the difference between the initial and the fully contracted area, expressed as a percentage. In the model, this variable is $100 \times\left\{(1+u(1, t))^{2}-(1+u(1, \infty))^{2}\right\} /$ $\left\{1-(1+u(1, \infty))^{2}\right\}$, where $u(1, \infty)$ is the limit of $u(1, t)$ as $t \rightarrow \infty$ which exists and is negative when collagen kinetics are neglected (Section 4). The simulated data are obtained from the numerical solutions of the axisymmetric model without collagen kinetics, with parameter values as in Fig. 3, except $\alpha=0.25$. The experimental data are extracted from measurements of contracting circular wounds in rat skin by McGrath \& Simon (1983). $t=0$ corresponds to day 3 in their experiments, since this is when the wound area begins to decrease, indicating the beginning of the proliferative phase of healing (Section 2).

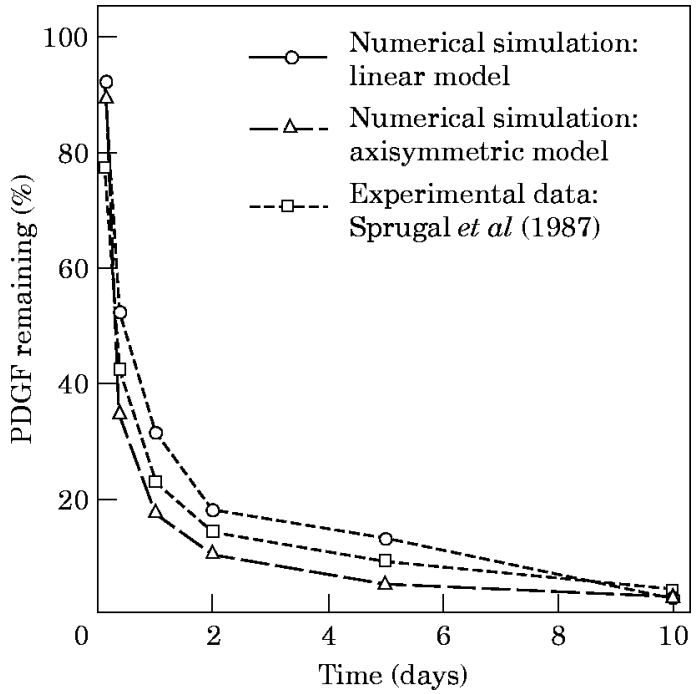

FIG. 5. Simulated and experimental data for the clearance of PDGF from the wound centre over a time course of 10 days. The chemical concentration at time $t$ is plotted as a percentage of the concentration at the onset of fibroplasia and contraction (for the model) or immediately after implantation (for the experimental subcutaneous wound chamber assay). The experimental data on PDGF clearance are derived from analyses of the contents of small porous "wound chambers" subcutaneously implanted into rats by Sprugel et al. (1987). For the model simulations, $100 \times c(0, t)$ is the appropriate variable, with parameter values as in Fig. 3. Data for the linear and axisymmetric models are shown alongside the experimental data because the long cylindrical shape of the implanted wound chambers is reminiscent of a combination of the two geometries. This is illustrated with remarkable clarity by the clearance curves shown above.

appears to be diffusion-driven, whereas the second, low-level decay may be partly due to production of the chemical by the cells which are infiltrating the wound.

\subsection{THE PROBLEM OF THE PERMANENCE OF WOUND CONTRACTION: AN EXPLANATION}

The model predicts that the contracted state is a transient (albeit long) phenomenon, in contrast to real wound healing, in which a permanently contracted state is normally observed.

To address this problem, we reconsider the collagen kinetics. At the dermal steady state, the collagen concentration, $\rho$, is set to unity by the kinetic terms in (9). If these terms are neglected, however, then (9) simply reduces to a convection equation for $\rho$. This approximation is validated by the relevant parameter values [notably $\omega$ and $\delta_{p}$-see (9), Appendix and Fig. 3 legend] and corroborated by the numerical simulations [Fig. 3(d)]. Consequently, when $n, m$ and $c$ assume their dermal steady state values, $\rho$ is not specified and thus depends on the initial conditions and on the subsequent healing dynamics. In particular, $\rho(x, t)$ may therefore evolve to a spatially 
inhomogeneous steady profile. From (10), the steady-state displacement is given by the equation:

$$
\begin{gathered}
\frac{\mathrm{d}^{2} u}{\mathrm{~d} x^{2}}+v \frac{\mathrm{d}}{\mathrm{d} x}\left(\frac{\rho}{\psi^{2}+\rho^{2}}\right)-s \rho u=0, \quad u(0)=0, \\
\frac{\mathrm{d} u}{\mathrm{~d} x}(\infty)=0 .
\end{gathered}
$$

The steady force balance equation (12) now contains a traction stress term (absent from (11) because there the collagen kinetics fix $\rho=1$ ). Thus, an important result is deduced: a non-zero steady-state displacement profile is possible if the collagen kinetics are neglected. In this case, the variables evolve over the 30-day time course in a similar manner to the simulations shown in Fig. 3. Over several years, however, the collagen concentration, $\rho$, remains very close to its 30 -day profile and does not return to its uniform, dermal value. Moreover, the displacement, $u$, having exhibited contraction over the 30-day time scale, does not relax back to zero, but remains permanently contracted, as demonstrated in Fig. 6. Equivalent results are inferred from the axisymmetric formulation (not shown).

Together, these results are consistent with the biology of wound healing because fibroplasia and contraction occur when the wound is in a transient, proliferative phase of the healing process. On the much longer time scale of remodelling (Section 2), a number of other processes are involved (Clark, 1988; Mast, 1992) that are deliberately excluded from the model. Thus, the model correctly predicts the permanence of wound contraction on the time scale over which it is intended to be applied.

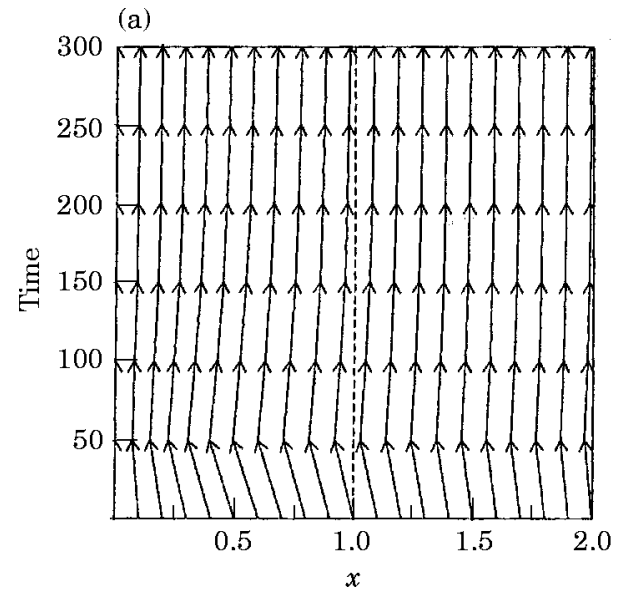

\section{Summary and Discussion}

The framework, functional forms (Section 3) and parameter value estimates (see Appendix) for the model presented in this paper are derived from a detailed examination of experimental and theoretical reports on fibroplasia and wound contraction.

The novel inclusion of two cell types, myofibroblasts and fibroblasts, enables careful examination of the specialized and distinct roles of these cells in fibroplasia and wound contraction (Section 2) and in pathological disorders of these processes. Explicitly incorporating the variable dynamics of a generic chemical which regulates the cellular healing responses is another novel aspect of the model that is evidently justified (Section 2).

These detailed considerations are central in the ability of the model to elicit a biologically realistic range of steady states (Section 4.1) representing unwounded dermis, a contracted wound and a variety of fibrocontractive disease states, some of which feature elevated cell densities only (see Table 1 in Section 4.1), whilst others exhibit considerably amplified cell, chemical and collagen levels, suggestive of chronic tissue pathologies. These are consistent with the variety of fibrocontractive diseases that are increasingly well characterised clinically, although the underlying pathogenic mechanisms remain poorly understood (Skalli \& Gabbiani, 1988; Rudolph \& Vande Berg, 1991; Rudolph et al., 1992; Asmussen \& Söllner, 1993; Murray, 1993). The spatiotemporal nature of the pathogenesis, evolution, cessation and regression of these disease states is currently under investigation (L. Olsen et al., submitted).

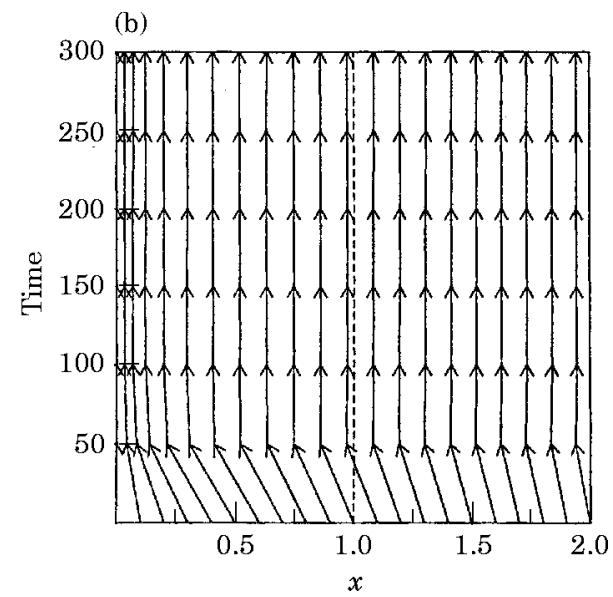

FIG. 6. Numerical simulations of the linear wound model, using the method of lines and Gear's method, showing the movement of tattoo marks to highlight tissue displacement, (a) as in Fig. 3(f), and (b) without kinetics for $\rho(x, t)$. Parameter values as in Fig. 3. The maximum time shown, $t=300$, corresponds to a dimensional time of approximately ten months. 
The existence and linear stability of these steady states are given by specific inequalities in terms of the model parameters (Section 4.1), which together with the detailed parameter value estimates proposed (see Appendix), yield a comprehensive predictive method of determining the occurrence and nature of these states.

The results predict that the system evolves to a steady state which is reminiscent of normal dermis in terms of the cellular and chemical levels but with a contracted steady displacement profile, if and only if the kinetics for collagen are neglected (Section 4). This is biologically appropriate in that collagen kinetics are fundamental to the matrix remodelling phase of healing, operating on a relatively long time scale, rather than to fibroplasia and contraction which are constitutive features of the transient, proliferative phase, for which the model is designed.

Qualitatively, the numerical simulations corroborate the normally observed processes of fibroplasia and wound contraction in the healing of real and experimental wounds (Sections 2 and 4). The linear and axisymmetric models are investigated, in which the wound domain is described in terms of one spatial coordinate (Fig. 2, Section 3). The qualitative model predictions are analogous in the two geometries, notably in the dependence on collagen kinetics of permanently contracted state and in the form of the displacement profiles obtained.

Quantitatively, the numerical results are similar for the two wound geometries, with relatively enhanced healing responses (using the same parameter values) towards the wound centre in the axisymmetric case compared to the linear case, in closer agreement with experimental data. This improved accuracy is expected, given the prevalence of circular wounds in experimental studies from which many parameter value estimates are inferred (see Appendix). Some numerical results exhibit excellent correlation with experimental data, however, as exemplified by the "biphasic" clearance of the chemical from the wound and by the reduction in the wound area due to contraction (details of which are provided in Section 4.4). We reiterate that quantitative discrepancies between the experimental and the model results may arise due to significant variations in healing characteristics between different species. A probable explanation is that many parameter values are estimated using data from a variety of in vitro and in vivo investigations using several species, including rats, pigs and humans. The model can only predict, therefore, "averaged mammalian" fibroplasia and wound contraction.
Another quantity that could be usefully compared with experimental data is the ratio of myofibroblasts to total cells in the model, $m /(n+m)$, over the time-course of contraction. Rudolph (1979) measured this figure for excisional wound healing in pigs and rats and McGrath \& Hundahl (1982) determined this ratio for a range of spatial locations in pig wounds. But even when the data are plotted so that the time axis is expressed as a percentage of the total time from the beginning to the end of wound contraction (in a particular species or in the model), it is evident that large qualitative disparities exist between different species (Rudolph, 1979; McGrath \& Hundahl, 1982). Therefore, comparing numerical solutions of the model with experimental results for this quantity is not reasonable.

The model results are not sensitive to small variations in any of the parameter values or initial conditions, provided points in parameter space are not varied near bifurcation subspaces or singular phenomena (see Appendix).

An important observation from the model is that dynamic cell and collagen gradients provide the driving force for wound contraction (Section 4). In this, myofibroblasts are responsible for effectively amplifying and transmitting the fibroblast-derived traction forces through the tissue, and the collagen matrix is a critical regulator.

L. O. expresses gratitude to the Engineering and Physical Sciences Research Council of Great Britain for a Research Studentship award to support this work. Part of this work was carried out while P.K.M. was visiting the School of Mathematics and Statistics, University of Sydney, Australia, and the Department of Mathematics, Williams College, Massachusetts, USA. This work was supported in part by a grant from the London Mathematical Society. We thank F. Arnold, J. Cook, P. Traqui, G. Cruywagen and J. D. Murray for helpful discussions.

\section{REFERENCES}

Alberts, B., Bray, D., Lewis, J., RafF, M., Roberts, K. \& Watson, J. D. (1989). The Molecular Biology of the Cell. New York: Garland Publ. Inc.

Arnold, F. \& WeSt, D. C. (1992). Angiogenesis in wound healing. Pharmac. Ther. 52, 407-422.

Asmussen, P. D. \& Söllner, B. (1993) Wound Care. Principles of Wound Healing. Hamburg: Beiersdorf medical Bibliothek.

Azzarone, B., Failly-Crepin, C., Daya Grosjean, L., Chaponnier, C. \& Gabbiani, G. (1983). Abnormal behaviour of cultured fibroblasts from nodule and nonaffected aponeurosis of Dupuytren's disease. J. Cell. Physiol. 117, 353-361.

BARD, J. B. L. \& HAY, E. D. (1975). The behaviour of fibroblasts from the developing avian cornea. Morphology and movement in situ and in vitro. J. Cell. Biol. 67, 400-418.

Barrow, G. M. (1981). Physical Chemistry for the Life Sciences. New York: McGraw-Hill Inc. 
BAuER, E. A. \& Uitto, J. (1982). Special tissue collagen: Skin. In: Collagen in Health and Disease (M. Jayson \& J. Weiss, eds) pp. 474-487. Edinburgh: Churchill Livingstone.

Bell, E., Ivarsson, B. \& Merrill, C. (1979). Production of a tissue-like structure by contraction of collagen lattices by human fibroblasts of different proliferative potential in vitro. Proc. natn. Acad. Sci. U.S.A. 76, 1274-1278.

Bowen-Pope, D. F., Malpass, T. W., Foster, D. M. \& Ross, R. (1984). Platelet-derived growth factor in vivo: Levels, activity and rate of clearance. Blood $\mathbf{6 4}, 458-469$.

Clark, R. A. F. (1988). Overview and general considerations of wound repair. In: The Molecular and Cellular Biology of Wound Repair (R. A. F. Clark \& P. M. Henson, eds) pp. 3-34. New York: Plenum.

Clark, R. A. F. (1989). Wound repair. Curr. Opin. Cell Biol., 1, 1000-1008.

Clark, R. A. F. (1991). Growth factors and wound repair. J. Cell. Biochem., 46, 1-2.

Clark, R. A. F. (1993). Regulation of fibroplasia in cutaneous wound repair. Am. J. Med. Sci. 306, 42-48.

Dover, R. \& Wright, N. A. (1991). The cell proliferation kinetics of the epidermis. In: Physiology, Biochemistry and Molecular Biology of the Skin (L. A. Goldsmith, ed.) pp. 239-265. New York: Oxford University Press.

Ehrlich, H. P. \& Rajaratnam, J. B. M. (1990). Cell locomotion forces versus cell contraction forces for collagen lattice contraction: An in vitro model of wound contraction. Tiss. Cell. 22, 407-417.

FunG, Y. C. (1981) Biomechanics. New York: Springer-Verlag.

Gabbiani, G. (1992). The biology of the myofibroblast. Kidney Int., 41, 530-532.

Gabbiani, G., Majno, G. \& Ryan, G. B. (1973). The fibroblast as a contractile cell: The myofibroblast. In: Biology of the Fibroblast (J. Pikkarainen \& K. Kulonen, eds) pp. 139-154. New York: Academic.

Gabbiani, G., Ryan, G. B. \& Majno, G. (1971). Presence of modified fibroblasts in granulation tissue and their possible role in wound contraction. Experientia 27, 549-550.

Grotendorst, G. R. (1992). Chemoattractants and growth factors. In: Wound Healing: Biochemical and Clinical Aspects (I. K. Cohen, R. F. Diegelmann, \& W. J. Lindblad, eds) pp. 237-246. Philadelphia: Saunders.

Huang, J. S., Huang, S. S. \& Deuel, T. F. (1984). Specific covalent binding of platelet-derived growth factor to human plasma $\alpha_{2}$-macroglobulin. Proc. natn. Acad. Sci. U.S.A. 81, 342-346.

Huang, J. S., Olsen, T. J. \& Huang, S. S. (1988). The role of growth factors in tissue repair I. Platelet-derived growth factor. In: The Molecular and Cellular Biology of Wound Repair (R. A. F. Clark \& P. M. Henson, eds) pp. 243-251. New York: Plenum.

Jefrerey, J. J. (1992). Collagen degradation. In: Wound Healing: Biochemical and Clinical Aspects (I. K. Cohen, R. F. Diegelmann \& W. J. Lindblad, eds) pp. 177-194. Philadelphia: Saunders.

Jennings, R. W. \& Hunt, T. K. (1992). Overview of postnatal wound healing. In: Fetal Wound Healing (N. S. Adzick \& M. T. Longaker, eds) pp. 25-52. New York: Elsevier.

Landau, L. D. \& Lifshitz, E. M. (1970). Theory of Elasticity. New York: Pergamon.

Lepistö, J., LaAto, M., Ninikoski, J., Lundberg, C., Gerdin, B. \& Heldin, C.-H. (1992). Effects of homodimeric isoforms of platelet-derived growth factor (PDGF-AA and PDGF-BB) on wound healing in rat. J. Surg. Res. 53, 596-601.

Longaker, M. T., Adzick, N. S. \& Ehrlich, H. P. (1992). Fetal wound contraction. In: Fetal Wound Healing (N. S. Adzick \& M. T. Longaker, eds) pp. 149-160. New York: Elsevier.

Martin, P., Hopkinson-Woolley, J. \& McCluskey, J. (1992). Growth factors and cutaneous wound repair. Prog. Gro. Fact. Res. 4, 24-44.

Mast, B. A. (1992). The skin. In: Wound Healing: Biochemical and Clinical Aspects (I. K. Cohen, R. F. Diegelmann \& W. J. Lindblad, eds) pp. 344-355. Philadelphia: Saunders.
McGrath, M. H. \& Hundahl, S. A. (1982). The spatial and temporal quantification of myofibroblasts. Plast. Reconstr. Surg. 69, 975-983.

McGrath, M. H. \& Simon, R. H. (1983). Wound geometry and the kinetics of wound contraction. Plast. Reconstr. Surg. 72, 66-72.

Morgan, C. J. \& Pledger, W. J. (1992). Fibroblast proliferation. In: Wound Healing: Biochemical and Clinical Aspects (I. K. Cohen, R. F. Diegelmann \& W. J. Lindblad, eds) pp. 63-76. Philadelphia: Saunders.

Murray, J. C. (1993). Scars and keloids. Dermatol. Clin. 11, 697-708.

Murray, J. D. (1989). Mathematical Biology. New York: Springer-Verlag.

Murray, J. D., Maini, P. K. \& Tranquillo, R. T. (1988) Mechanochemical models for generating biological pattern and form in development. Phys. Rep. 171, 59-84.

Murray, J. D. \& Oster, G. F. (1984). Cell traction models for generating pattern and form in morphogenesis. J. math. Biol. 19, 265-279.

Murray, J. D., Oster, G. F. \& Harris, A. K. (1983). A mechanical model for mesenchymal morphogenesis. J. math. Biol. 17, 125-129.

ODLAND, G. \& Ross, R. (1968). Human wound repair: I. Epidermal regeneration. J. Cell Biol. 39, 135-151.

OdLAND, G. F. (1991). Structure of the skin. In: Physiology, Biochemistry and Molecular Biology of the Skin (L. A. Goldsmith, ed) pp. 3-62. New York: Oxford University Press.

Oster, G. F., Murray, J. D. \& Harris, A. K. (1983). Mechanical aspects of mesenchymal morphogenesis. J. Embryol. expl Morphol. 78, 83-125.

Pierce, G. F., Brown, D. \& Mustoe, T. A. (1991a). Quantitative analysis of inflammatory cell influx, procollagen type I synthesis, and collagen cross-linking in incisional wounds: Influence of PDGF-BB and TGF- $\beta_{1}$ therapy. J. Lab. Clin. Med., 117, 373-382.

Pierce, G. F., Vande Berg, J., Rudolph, R., Tarpley, T. \& Mustoe, T. A. (1991b). Platelet-derived growth factor-BB and transforming growth factor betal selectively modulate glycosaminoglycans, collagen, and myofibroblasts in excisional wounds. Am. J. Pathol. 138, 629-646.

Raines, E. W., Bowen-Pope, D. F. \& Ross, R. (1990). Platelet-derived growth factor. In: Handbook of Experimental Pharmacology, Vol. 95 part I: Peptide Growth Factors and their Receptors (M. B. Sporn \& A. B. Roberts, eds) pp. 173-262. Heidelberg: Springer-Verlag.

Roberts, A. B., Sporn, M. B., Assoian, R. K., et al. (1986). Transforming growth factor type $\beta$ : Rapid induction of fibrosis and angiogenesis in vivo and stimulation of collagen formation in vitro. Proc. natn. Acad. Sci. U.S.A. 83, 4167-4171.

Ross, R., Raines, R. W. \& Bowen-Pope, D. F. (1986). The biology of platelet-derived growth factor. Cell 46, 155-169.

RUDOLPH, R (1979). Location of the force of wound contraction. Surg. Gynecol. Obstet. 148, 547-551.

RudolPh, R. (1980). Contraction and the control of contraction. World J. Surg. 4, 279-287.

Rudolph, R., Vande Berg, J. \& Ehrlich, H. P. (1992). Wound contraction and scar contracture. In: Wound Healing: Biochemical and Clinical Aspects (I. K. Cohen, R. F. Diegelmann \& W. J. Lindblad, eds) pp. 96-114. Philadelphia: Saunders.

RudolPh, R. \& VANDE BerG, J. (1991). The myofibroblast in Dupuytren's contracture. Hand Clin. 7, 683-692.

Sato, N., Beitz, J. G., Kato, J., Yamamoto, M., Clark, J. W., Calabresi, P. \& Frackelton, Jr. A. R. (1993). Platelet-derived growth factor indirectly stimulates angiogenesis in vitro. Am. J. Pathol. 142, 1119-1130.

Seppä, H., Grotendorst, G., Seppä S., Schiffmann, E. \& Martin, G. R. (1982). Platelet-derived growth factor is chemotactic for fibroblasts. J. Cell. Biol. 92, 584-588.

Shah, M., Foreman, D. M. \& Ferguson, M. W. J. (1992). Control of scarring in adult wounds by neutralising antibody to transforming growth factor $\beta$. Lancet 339, 213-214. 
Sherratt, J. A., Martin, P., Murray, J. D. \& Lewis, J. (1992). Mathematical models of wound healing in embryonic and adult epidermis. IMA J. Math. Appl. Med. Biol. 9, 177-196.

Sherratt, J. A. \& Murray, J. D. (1992). Epidermal wound healing: The clinical implications of a simple mathematical model. Cell Transpl. 1, 365-371.

Sherratt, J. A., Sage, E. H. \& Murray, J. D. (1993). Chemical control of eukaryotic cell movement: A new model. $J$. theor. Biol. 162, 23-40.

Singer, I. I., Kawka, D. W., Kazais, D. M. \& Clark, R. A. F. (1984). In vivo co-distribution of fibronectin and actin fibers in granulation tissue: Immunofluorescence and electron microscope studies of the fibronexus at the myofibroblast surface. J. Cell. Biol. 98, 2091-2106.

Skalli, O. \& Gabbiani, G. (1988). The biology of the myofibroblast. Relationship to wound contraction and fibrocontractive diseases. In: The Molecular and Cellular Biology of Wound Repair (R. A. F. Clark \& P. M. Henson, eds) pp. 373-402. New York: Plenum.

Skalli, O., Schürch, W., Seemayer, T., Lagacé, R., Montandon, D., Pittet, B. \& Gabbiani, G. (1989). Myofibroblasts from diverse pathologic settings are heterogeneous in their content of actin isoforms and intermediate filament proteins. Lab. Invest. 60, 275-285.

SNowden, J. M. \& ClifF, W. J. (1985). Wound contraction. Correlations between the tension generated by granulation tissue, cellular content and rate of contraction. Q.J. expl. Physiol. 70, 539-548.

Sprugel, K. H., McPherson, J. M., Clowes, A. W. \& Ross, R. (1987). Effects of growth factors in vivo. Am. J. Pathol. 129, 601-613.

Stopak, D. \& Harris, A. K. (1982). Connective tissue morphogenesis by fibroblast traction: I: Tissue culture observations. Dev. Biol. 90, 383-398.

Tranquillo, R. T. \& Murray, J. D. (1992). Continuum model of fibroblast-driven wound contraction: Inflammation-mediation. J. theor. Biol. 158, 135-172.

Uiтto, J. (1984). Interstitial collagens. In: Biology of the Integument, Vol. 2: Vertebrates (J. Bereiter-Hahn, A. G. Matoltsky \& K. S. Richards, eds) pp. 800-809. Berlin: Springer-Verlag.

Vande Berg, J. S., Rudolph, R., Poolman, W. L. \& Disharoon, D. R. (1989). Comparative growth dynamics and actin concentration between cultured human myofibroblasts from granulating wounds and dermal fibroblasts from normal skin. Lab. Invest. 61, 532-538.

Whalen, G. F. \& Zetter, B. R. (1992). Angiogenesis. In: Wound Healing: Biochemical and Clinical Aspects pp. 77-95. Philadelphia: Saunders.

\section{APPENDIX}

\section{PARAMETER ESTIMATES AND NONDIMENSIONALIZATION}

In view of the large number of parameters in the model [Section 3: see eqns (1-5)], accurate parameter value estimation is a vital part of the modelling process. An important step in this process is to reduce the number of dimensional parameters by the following simplifying assumptions:

- The half-maximal concentrations for the selfregulated production and consumption of the chemical are equal, since the same cell surface interaction mechanism occurs in the two cases, and we set $\Gamma_{p}=\Gamma_{c}=: \Gamma$.
- The ratio of the roles of myofibroblasts to fibroblasts in the production of the chemical is equal to that in the consumption of the chemical, so $\zeta_{p}=\zeta=: \zeta$.

- A similar argument for the roles of the two cell types in collagen biosynthesis and degradation yields $\eta_{b}=\eta_{d}=: \eta$.

For the remaining parameters, we first estimate values for the typical scalings which are used to nondimensionalize the variables (see below).

$L$ : A typical length scale of an excisional dermal wound is $1 \mathrm{~cm}$.

$T$ : The progress of a typical wound through the inflammatory and proliferative phases of healing (Section 2) is normally measured in days, so we set $T=1$ day.

$n_{0}$ : Human dermal fibroblasts generally have dimensions of the order $100 \mu \mathrm{m} \times 100 \mu \mathrm{m} \times 10 \mu \mathrm{m}$. Furthermore, fibroblasts are sparse in normal adult dermis (Morgan \& Pledger, 1992), and stipulating that there are, on average, ten cells per cubic millimetre, yields the value $n_{0}=10^{4} \mathrm{ml}^{-1}$.

$c_{0}$ : PDGF is stored within platelets in the bloodstream at low concentrations-reports suggest $15-50 \mathrm{ng} \mathrm{ml}^{-1}$ (Huang et al., 1988). The concentrations released at the wound by platelets and by activated inflammatory cells should not, therefore, exceed these levels, and we propose $c_{0}=10 \mathrm{ng} \mathrm{ml}^{-1}$. The initial chemical concentration profile is $c_{\text {init }}(x)=c_{0}\left(1+x^{4}\right)^{-1}$.

$\rho_{0}$ : The density of the human body is similar to that of water, namely $1 \mathrm{~g} \mathrm{ml}^{-1}$. By mass, $1 \mathrm{ml}$ of tissue contains approximately $70 \%$ water, $15 \%$ fat and $15 \%$ other substances, of which roughly three-quarters is collagen in the case of dermal tissue (Bauer \& Uitto, 1982). Hence, $\rho_{0} \approx 0.1 \mathrm{~g} \mathrm{ml}^{-1}$ is a valid order of magnitude estimate.

With these typical scales, the following nondimensional variables are introduced:

$$
\begin{aligned}
& \tilde{x}=\frac{x}{L}, \quad \tilde{t}=\frac{t}{T}, \quad \tilde{n}=\frac{n}{n_{0}}, \quad \tilde{m}=\frac{m}{n_{0}}, \quad \tilde{c}=\frac{c}{c_{0}}, \\
& \tilde{\rho}=\frac{\rho}{\rho_{0}}, \quad \tilde{u}=\frac{u}{L} .
\end{aligned}
$$

Turning to the dimensional model parameter values: $D_{n}$ : Electron microscope studies of migrating human fibroblasts suggest that the maximum rate of random motility is approximately $1 \mu \mathrm{m} \mathrm{min}^{-1}$ (Bard \& Hay, 1975), yielding a diffusion coefficient of $1.7 \times 10^{-10} \mathrm{~cm}^{2} \mathrm{~s}^{-1}$.

$a_{n}$ : In the absence of reported quantitative studies to determine the chemotactic parameters for dermal fibroblasts, we conducted numerical simulations for a 
range of values of $a_{n}$. Because fibroplasia fundamentally depends on inflammatory stimuli [11], we suggest that the chemotactic flux of fibroblasts into the wound site should predominate over the random diffusive flux and therefore set $a_{n}=5 \mathrm{ng} \mathrm{cm}^{-1} \mathrm{day}^{-1}$. $b_{n}$ : Concentrations as low as $1 \mathrm{ng} \mathrm{ml}^{-1}$ of PDGF elicit significant fibroblast chemotaxis (Seppä et al., 1982), and experimental data indicate that the concentration for the half-maximal response is about $2 \mathrm{ng} \mathrm{ml}^{-1}$ (Grotendorst, 1992).

$r_{n}, r_{n_{\max }}, K$ and $d_{n}$ : The basal growth rate, $r_{n}$, of a population of fibroblasts is difficult to determine experimentally because the population appears to be constant, for the following reason: when $m=0$ and $c=0$, there is a steady-state cell density, $n^{*}$, given by the equation: $r_{n} n^{*}(1-n / K)-d_{n} n^{*}=0$, implying either $n^{*}=0$ or $n^{*}=K\left(1-d_{n} / r_{n}\right)$. Because $m=0$ and $c=0$ are characteristic of normal dermis, we assume that $n^{*}=n_{0}$, the normal dermal fibroblast density. Thus $r_{n}$ may be determined once $K$ and $d_{n}$ are known.

The maximal fibroblast density, $K$, is the density at which cell proliferation is totally inhibited. Experimental growth curves and various studies using high-density fibroblast populations indicate a value of the order of $10^{6}$ cells per millimetre, so we take $K=10^{6} \mathrm{ml}^{-1}$. As a check, this implies that each fibroblast occupies a volume of at least $(100 \mu \mathrm{m})^{3}$, which is consistent given that the maximum linear dimension of a fibroblast is about $100 \mu \mathrm{m}$ ).

Cultured dermal fibroblasts have an average lifespan of approximately 40 population doublings (Azzarone et al., 1983), so denoting the maximal doubling time by $T_{2}$ yields $d_{n}=(\ln 2) /\left(40 \times T_{2}\right)$. Estimates for $T_{2}$ are typically around $18 \mathrm{~h}[1,32]$, which implies $d_{n} \approx 0.02$ day $^{-1}$. Hence, $r_{n}=d_{n}\left(1-n_{0} /\right.$ $K)^{-1} \approx 0.99 d_{n}=0.0198$ and $r_{n_{\max }}=\left(\ln 2 / T_{2}\right)-r_{n} \approx 0.9$. $C_{1 / 2}$ : The chemical concentrations required to enhance fibroblast proliferation are somewhat higher than those for chemotactic responses (Grotendorst, 1992; Lepistö et al., 1992). Experimental evidence indicates that half-maximal enhancement corresponds to concentrations of about $10 \mathrm{ng} \mathrm{ml}^{-1}$ (Grotendorst, 1992).

$k_{1_{\max }}, C_{k}$ and $k_{2}$ : Quantitative data on the rates of conversion between fibroblasts and myofibroblasts are not available, so the following intuitive assumptions are proposed: first, the disappearance of myofibroblasts at the end of contraction is predominantly due to reversion to the fibroblast phenotype rather than cell death, so $k_{2}>d_{m}$; second, the "half-life" of the myofibroblast to fibroblast conversion is less than a day (this process primarily involves intracellular modifications, so the distances involved are very small and signals are transmitted quickly).
This half-life is $(\ln 2) / k_{2}$, so we take $k_{2} \approx 1$ day $^{-1}$; third, given the intracellular growth factor-driven events involved, the maximal rate of conversion of fibroblasts to myofibroblasts is faster than the reverse process, so we take $k_{1_{\max }} \approx 10 \mathrm{day}^{-1}$; finally, the relatively low chemical concentrations in the wound should stimulate myofibroblast production, so a reasonable order-of-magnitude estimate is $C_{k}=1 \mathrm{ng} \mathrm{ml}^{-1}$.

$\epsilon_{r}$ : Growth curves of myofibroblasts cultured from contracting wounds indicate that the growth rate is lower $(50-100 \%)$ than for normal dermal fibroblast cultures (Vande Berg et al., 1989; Rudolph \& Vande Berg, 1991). The variability in the experimental data is due to the heterogeneity of the cellular composition of wounds (Snowden \& Cliff, 1985; Skalli \& Gabbiani, 1988). In particular, the myofibroblast/ fibroblast density ratio varies with time and space during healing (McGrath \& Hundahl, 1982). Assuming that a population of "pure" myofibroblasts proliferates at half the rate of fibroblasts, we estimate $\epsilon_{r}=0.5$.

$\lambda$ : There is currently no data on death rates of myofibroblasts, qualitatively or quantitatively. Using the following inequalities: $d_{n}<d_{m}=\lambda d_{n}<k_{2}$ (derived from earlier estimates), however, and the values provided for the bounds, we obtain $1<\lambda<50$ and choose $\lambda=10$.

$D_{c}$ : Although direct experimental measurement of the diffusivity of PDGF is lacking, its molecular weight has been consistently recorded at around $30000 \mathrm{Da}$, from which the following theoretical argument can be used to estimate a value for $D_{c}$. For many large proteins, such as wound growth factors, in aqueous solution at a particular temperature and pressure, $D_{c} \propto M_{c}^{-1 / 3}$ is a good approximation (Barrow, 1981), where $M_{c}$ is the molecular weight of the chemical. The constant of proportionality is unknown, but if $c^{\prime}$ represents a second chemical, then $D_{c} \approx\left(M_{c^{\prime}} / M_{c}\right)^{1 / 2} D_{c^{\prime}}$. Data is available for epidermal growth factor, whose molecular weight is 6045 and diffusion coefficient is $9.75 \times 10^{-7} \mathrm{~cm}^{2} \mathrm{~s}^{-1}$. By the argument above, the estimated value of $D_{c}$ is $5.7 \times 10^{-7} \mathrm{~cm}^{2} \mathrm{~s}^{-1}$.

$p, k_{p}^{\prime}, k_{c}^{\prime}$ and $\Gamma$ : The functional forms for the production and consumption of the chemical in (3) involving these parameters are derived from the schematic reactions:

$$
R+C \stackrel{k_{p}^{\prime}}{\longrightarrow} R+p C \text { and } R+C \stackrel{k_{c}^{\prime}}{\longrightarrow} R
$$

respectively, where $R$ represents a free cell-surface receptor molecule, $C$ a chemical molecule, $p$ is an (unknown) positive integer and $k_{p}^{\prime}$ and $k_{c}^{\prime}$ are the rate 
constants. Denoting the concentrations of $R$ by $r$ and $C$ by $c$, then the law of mass action implies $\partial c / \partial t=(p-1) k_{p}^{\prime} r c-k_{c}^{\prime} r c$. Assuming that the concentration of bound receptors, $r^{\text {bound }}$, is a saturating monotonic increasing function of $c, r^{\text {bound }}=$ $r^{\text {total }} c /(\Gamma+c)$, say [see also Sherratt et al., (1993)], where the total receptor concentration is $r^{\text {total }}=$ $r^{\text {bound }}+r$, then $r=\Gamma r^{\text {total }} /(\Gamma+c)$. Finally, $r^{\text {total }}$ is proportional to cell density, $n$, giving the expressions in (3).

The only information on the values of these parameters is that they are positive. By considering the existence and linear stability of steady states (Section 4.1), bounds may be imposed on the value of a dimensionless parameter, $\kappa_{c}$, which is related to the dimensional quantity $(p-1) k_{p}^{\prime}-k_{c}^{\prime}$.

$\zeta$ : Myofibroblasts also express cell surface receptors for PDGF (Sato et al., 1993) implying that these cells also regulate the chemical concentration, so $\zeta>0$. Without evidence to suggest that myofibroblasts metabolize PDGF at a different rate of fibroblasts, we take $\zeta=1$.

$d_{c}$ : When excess PDGF is released from platelets and other stores, it quickly forms a complex with the plasma binding protein $\alpha_{2}$-macroglobulin with a half-life of $4 \mathrm{~min}$ (Huang et al., 1984, 1988). This bound complex is recognized by a receptor for $\alpha_{2}$-macroglobulin on the surface of liver cells, and is rapidly cleared from plasma with a half-life of only 2 min (Bouen-Pope et al., 1984), a figure which yields the estimate $d_{c}=500 \mathrm{day}^{-1}$. It remains unknown, however, whether (a) some of the chemical released during healing is unavailable for binding to $\alpha_{2}$-macroglobulin, for example if there are insufficient levels of the binding protein locally at the wound site, and (b) the bound PDGF- $\alpha_{2}$-macroglobulin complex may be recognized by fibroblasts and myofibroblasts and the PDGF subsequently internalized and metabolized. In these scenarios $d_{c}$ could be considerably smaller, with significant consequences for the model solutions.

$r_{\rho}, R_{\rho}$ and $d_{\rho}$ : As stated in Section 4.1., a uniform steady state $(n, m, c, \rho, u)=\left(n_{0}, 0,0, \rho_{0}, 0\right)$ exists, representing unwounded dermis. Therefore, if three of the parameters $r_{\rho}: R_{\rho}, d_{\rho}$ and $\rho_{0}$ are known, then so is the fourth, then so is the fourth, by the virtue of $r_{\rho}\left(R_{\rho}^{2}+\rho_{0}^{2}\right)^{-1}-d_{\rho} \rho_{0}=0$, from equation (4) in Section 3. The half-life of collagen turnover in the adult human dermis is 2.5 years (Uitto, 1984), which yields $d_{\rho}=(\ln 2) /\left(365 \times 2.5 \times n_{0}\right)$ day $^{-1} \approx 8 \times 10^{-8}$ day $^{-1}$. We estimate $R_{\rho}$ using the following intuitive argument. The sigmoidal biosynthesis inhibition function, $\left(R_{\rho}^{2}+\rho^{2}\right)^{-1}$, is half of its maximal (at $\left.\rho=0\right)$ value when $\rho=R_{\rho}$. We assume that this substantial downregula- tion of its production occurs at abnormally high collagen concentrations, so that $R_{\rho}>1$, but not so large that biosynthesis inhibition is essentially negligible. In the numerical simulations, we set $R_{\rho}=3 \rho_{0}$. Finally, $r_{\rho}$ is found by using the estimates for the other parameters in the above equation.

$r_{\rho_{\max }}$ : Studies using implanted dermal wound "chambers" have indicated that PDGF alone does not enhance collagen biosynthesis per cell (Lepistö et al., 1992). Transforming growth factor-beta (TGF- $\beta$ ), however, has been shown to markedly increase collagen synthesis per cell (Roberts et al., 1986; Pierce et al., 1991a, b). The synergistic effects of these two growth factors may accelerate collagen biosynthesis up to tenfold, so $r_{\rho_{\max }}=10 r_{\rho}$ is a reasonable estimate.

$C_{\rho}$ : Experimental data suggest that half-maximal enhancement of collagen biosynthesis occurs at TGF- $\beta$ concentrations of the order of $1 \mathrm{ng} \mathrm{ml}$ (Roberts et al., 1986), thus giving an estimate for $C_{\rho} . \eta$ : Cultured myofibroblasts are biosynthetically active (Skalli \& Gabbiani, 1988), implying that $\eta>0$, and observations have suggested that myofibroblasts secrete twice as much type I collagen as fibroblasts (Rudolph \& Vande Berg, 1991), so that $\eta=2$.

$\mu_{1}, \mu_{2}, E^{\prime}$ and $v^{\prime}$ : These mechanical parameters are related to the bulk and shear viscosities, Young's modulus and Poisson's ratio for the adult dermis; for which data is not readily available - a ubiquitous problem in mechanochemical models. Consequently, (dimensionless) parameter variation analyses were conducted numerically, from which approximate ranges of parameter values for which the model yields biologically and mathematically valid solutions were derived.

$\tau_{0}$ : The traction force per unit of collagen concentration and fibroblast cell density has been studied in detail both mathematically (Murray \& Oster, 1984; Tranquillo \& Murray, 1992) and experimentally (Snowden \& Cliff, 1985; Stepak \& Harris, 1982). Data has not been given, however, for cell traction forces in a three-dimensional collagen gel-a system which is currently attracting considerable research. At present, therefore, numerical experiments are again used to suggest order-of-magnitude estimates.

$\xi$ : Sharp myofibroblast enhancement of traction is proposed per unit of cell density, motivated by the strong correlation of myofibroblast density and the rate of contraction (Rudolph, 1979; McGrath \& Hundahl, 1982; Skalli \& Gabbiani, 1988), and we set $\xi=10 . \xi$ and $\tau_{0}$ are intimately related because their product is the coefficient of the 
myofibroblast-enhanced component of the cell traction term in the force balance equation (5).

$s$ : This parameter measures the strength of the linear restoring forces acting against tissue displacement, but quantitative data on the magnitude of these forces are not available. Therefore, acceptable parameter estimates were investigated numerically, in conjunction with the other parameters in the force balance equation, as above.

$R_{\tau}$ : The following arguments, based upon (a) the biomechanics of wound contraction and (b) the form of the cell traction stress term in the force balance equation, are used to estimate this parameter value. In vitro investigations using collagen gels have demonstrated that the rate of contraction is proportional to the cell density (Bell et al., 1979), which is consistent with the proposed traction function, since $\tau \propto n$. Further, the rate of contraction is inversely proportional to the collagen concentration (Bell et al., 1979). Significantly, $\tau \propto \rho^{-1}$ in the limit as $R_{\tau} \rightarrow 0$ for values of $\rho>R_{\tau}$. Thus the given form agrees with the experimental data of Bell et al. (1979), if $R_{\tau}$ is sufficiently small. Next, the mathematical consequences of the traction term are considered by writing:

$$
\tau(\rho)=\frac{\rho}{R_{\tau}^{2}+\rho^{2}}, \quad \text { where } \rho=\rho(x, t) .
$$

The momentum flux is given by the cell traction stress gradient:

$$
\frac{\partial \tau}{\partial x}=\frac{R_{\tau}^{2}-\rho^{2}}{\left(R_{\tau}^{2}+\rho^{2}\right)^{2}} \frac{\partial \rho}{\partial x} .
$$

As $x$ increases from zero, the wound centre, we expect $\rho$ to be a monotonically increasing function of $x$. Hence, $\partial \tau / \partial x$ and $R_{\tau}^{2}-\rho^{2}$ have the same sign. In order to exhibit wound contraction, the traction forces must act towards the wound centre, so the sign of $\partial \tau / \partial x$ must be negative. Thus, an approximate upper bound for $R_{\tau}$ is the minimum value of $\rho$ for $x>0$, so $R_{\tau}<\rho_{\text {init }}$. Without further guidance, we choose $R_{\tau}=0.5 \rho_{\text {init }}$. $\rho_{\text {init }}$ : The concentration of type I collagen in the wound at the onset of contraction is a small fraction of that of normal dermis, and in the absence of quantitative evidence from the literature, we set $\rho_{\text {init }}=0.01 \rho_{0}$.

Finally, the following nondimensionalization scheme is used to relate the parameters in the model equations $(6-10)$ to those in $(1-5)$ :

$$
\begin{aligned}
& \tilde{D}_{n}=\frac{D_{n} T}{L^{2}} \quad \tilde{\alpha}=\frac{a_{n} T}{L^{2} c_{0}} \quad \tilde{\beta}=\frac{b_{n}}{c_{0}} \quad \tilde{\sigma}=r_{n} T \quad \tilde{A}=\frac{r_{n_{\max }}}{r_{n}} \\
& \tilde{B}=\frac{C_{1 / 2}}{c_{0}} \quad \bar{\gamma}=\frac{n_{0}}{K} \quad \tilde{\kappa}_{1}=k_{1_{\max }} T \quad \tilde{C}=\frac{C_{k}}{c_{0}} \quad \tilde{\kappa}_{2}=k_{2} T \\
& \tilde{\delta}=d_{n} T \quad \tilde{\epsilon}_{\sigma}=\epsilon_{r} \quad \tilde{\lambda}=\frac{d_{m}}{d_{n}} \quad \tilde{D}_{c}=\frac{D_{c} T}{L^{2}} \\
& \tilde{\kappa}_{c}=\left((p-1) k_{p}^{\prime}-k_{c}^{\prime}\right) T n_{0} \\
& \tilde{\gamma}_{c}=\frac{\Gamma}{c_{0}} \quad \tilde{\zeta}=\zeta \quad \tilde{\delta}_{c}=d_{c} T \quad \tilde{\omega}=\frac{r_{\rho} R_{\rho}^{2} T n_{0}}{\rho_{0}^{3}} \quad P=\frac{r_{\rho_{\max }}}{r_{\rho}} \\
& \tilde{Q}=\frac{C_{\rho}}{c_{0}} \quad \tilde{\phi}=\frac{R_{\rho}}{\rho_{0}} \quad \tilde{\delta}_{\rho}=d_{\rho} T n_{0} \quad \tilde{\eta}=\eta \\
& \tilde{\mu}=\frac{\mu_{1}+\mu_{2}}{E^{\prime}\left(1+v^{\prime}\right) T} \\
& \tilde{v}=\frac{\tau_{0} R_{\tau}^{2} n_{0}}{E^{\prime}\left(1+v^{\prime}\right) \rho_{0}} \quad \tilde{\xi}=\xi n_{0} \quad \tilde{\psi}=\frac{R_{\tau}}{\rho_{0}} \quad \tilde{s}=\frac{s L^{2} \rho_{0}}{E^{\prime}\left(1+v^{\prime}\right)} \\
& \tilde{\rho}_{\text {init }}=\frac{\rho_{\text {init }}}{\rho_{0}} \quad \tilde{c}_{\text {init }}(x)=\frac{c_{\text {init }}(x)}{c_{0}}
\end{aligned}
$$

\section{SENSITIVITY OF THE PARAMETER ESTIMATES}

We remark that parameter variation sensitivity analyses were conducted numerically for all of the model parameters (using the dimensionless quantities). For the parameters whose values were estimated from experimental data or theoretical calculations, small variations in these values resulted only in small quantitative changes in the numerical solutions of the system. The remaining parameters, whose values were not so readily estimated, were chosen such that in parameter space, the system was not near a region of sensitive behaviour in the numerical simulations, such as bifurcation surfaces. Hence, all of the parameter values used for the results in this paper (see Fig. 3 legend) required relatively large deviations in order to elicit significantly altered numerical results. 